\title{
Coupling of thermocapillary convection and evaporation effect in a liquid layer when the evaporating interface is open to air
}

\author{
ZHU ZhiQiang \& LIU QiuSheng* \\ Key Laboratory of Microgravity (National Microgravity Laboratory), Institute of Mechanics, Chinese Academy of Sciences, Beijing 100190, \\ China
}

Received March 9, 2009; accepted June 29, 2009

\begin{abstract}
The coupling mechanism of thermocapillary convection and evaporation effect in evaporating liquids was studied experimentally. The experiments were carried out to study a thin evaporating liquid layer in a rectangular test cell when the upper surface was open to air. By altering the imposed horizontal temperature differences and heights of liquid layers, the average evaporating rate and interfacial temperature profiles were measured. The flow fields were also visualized by PIV method. For comparison, the experiments were repeated by use of another two non-evaporating liquids to study the influence of evaporation effect. The results reveal evidently the role that evaporation effect plays in the coupling with thermocapillary convection.
\end{abstract}

thermocapillary convection, evaporation, temperature profile, flow pattern

Citation: Zhu Z Q, Liu Q S. Coupling of thermocapillary convection and evaporation effect in a liquid layer when the evaporating interface is open to air. Chinese Sci Bull, 2010, 55: 233-238, doi: 10.1007/s11434-009-0693-2

The research of thermocapillary convection in a fluid layer subjected to a horizontal temperature gradient has given rise to more fundamental and practical interests, due to the great applications of which in many technological and industrial fields such as solidification processes in crystal growth, laser welding and thin-film coating. Over the last few decades, most of the studies have been focused on the instabilities of thermocapillary convection in the float-zone, the transition of which to oscillatory flow was regarded to relate to the flaw of the resulting crystal $[1,2]$. Subsequently, Smith and Davis $[3,4]$ theoretically studied the instabilities of pure thermocapillary convection with planar interface, i.e. the externally imposed temperature gradients being parallel to the interface. Succeeding theoretical and experimental work extended the study area to the combined buoyancythermocapillary convection. Researchers not only validated the theory of Smith and Davis. Meanwhile, they developed varieties of methods to find some new phenomena and

*Corresponding author (email: liu@imech.ac.cn) mechanisms [5-11]. Nevertheless, the studies on the thermocapillary convection have been relatively mature.

Previous work only involved the research of pure thermocapillary convection or combined thermocapillary-buoyancy convection. With the deepening of scientific research, especially the development of space industry, the interdisciplinary researches of thermocapillary convection and other subjects have attracted more and more attentions, for example, the study of the evaporation effect coupling with thermocapillary convection, which plays a very important role in many industrial applications such as nuclear reactor, thin-film evaporator and heat pipe in space. In the research of material fields, efforts involving the study of evaporation effect have already been made to investigate evaporation phenomena $[12,13]$. However, only a few studies introduced evaporation into thermocapillary convection. Actually, the coupling mechanisms between evaporation and convection are very complicated. Some hardly unbeknown experiment results were found in the study of Marangoni-Bénard convection in an evaporating liquid layer $[14,15]$, i.e. the insta- 
bilities induced by the vertical temperature gradients. Subsequently, a series of numerical work has been performed to explain the mechanisms of the new results $[16,17]$. As to the analysis of thermocapillary convection coupling with evaporation effect in a horizontal thin liquid layer, only preliminary numerical study was performed [18]. Recently, in a special experimental environment, researchers found a big temperature jump at a spherical evaporating interface $[19,20]$. Yet there are no relevant papers regarding more common experiments in a plane thin evaporating layer.

Anyhow, the combining mechanism of evaporation effect and thermocapillary convection is more complicated, and needs comprehensive understanding. Motivated by the description above, we performed a series of experiments to study the coupling of evaporation and thermocapillary convection, the process of heat and mass transfer at the interface of a planar thin liquid layer with an evaporating phase change.

\section{Experimental apparatus and procedure}

Our experiments were designed to make some measurements of thermocapillary convection in an evaporating liquid layer. Schematic diagram of the experimental apparatus is shown in Figure 1. The test cell is a rectangular container constructed by two aluminium blocks at opposite sides and two pieces of optical glass placed perpendicularly to the aluminium blocks. The cell has a width of $0.04 \mathrm{~m}$ along the temperature gradient, and a length of $0.08 \mathrm{~m}$ perpendicular to the temperature gradient, and were kept constant in all the experiments. The bottom of the container is a piece of optical glass for thermal insulation. Before each experiment, the bottom was regulated to the level position.

The horizontal temperature difference $\left(\Delta T=T_{\mathrm{h}}-T_{\mathrm{c}}\right)$ was achieved through the temperature control of the two alu- minium end walls. The cold wall was thermally controlled by the refrigerated circulator with an accuracy of $0.02^{\circ} \mathrm{C}$. An electrical heater was attached on the outer side of the hot wall, and the temperature was controlled by means of PID loops with an accuracy of $0.05^{\circ} \mathrm{C}$. In order to measure $T_{\mathrm{h}}$ and $T_{\mathrm{c}}$, four thermocouples were mounted at the inner of the two aluminium walls. Note that the two aluminium blocks were coated with insulated material. In addition, the whole of the test cell was placed in a Plexiglas box to minimize the disturbance of the environmental temperature and air flow.

To measure the evaporating rates of the liquid in the cell, a laser co-focal displacement meter was used during the experiments. The laser beam emitted from the displacement meter could be focused to track the local surface of the liquid layer with an accuracy of $0.3 \mu \mathrm{m}$. Therefore, we could obtain the average evaporating rate by calculating the change of the interface height in case of stationary flow.

One aim of the experiments is to obtain the temperature profiles along the interface when the horizontal temperature gradient was achieved. Temperature measurements were performed using a thermocouple with a diameter of $50 \mu \mathrm{m}$ and a response time of $0.02 \mathrm{~s}$. The thermocouple was mounted at a $X Y Z$ translation stage to ensure movements in three directions. The temperature data of the liquid layer, together with $T_{\mathrm{h}}$ and $T_{\mathrm{c}}$, were acquired by a data acquisition system, which could change the recording intervals. A computer programmed and controlled the translation stage and the data acquisition system to obtain temperature data at different spatial and temporal intervals.

Flow visualization was accomplished by the PIV technique. A $1 \mathrm{~mm}$ thick laser sheet illuminated a streamwise transverse plane of the liquid layer. Tracer particles with a diameter of $5 \mu \mathrm{m}$ were seeded in the illuminated plane to reflect the flow field. A CCD camera was used to record the images of the motion of the particles from the front glass wall of the test cell.
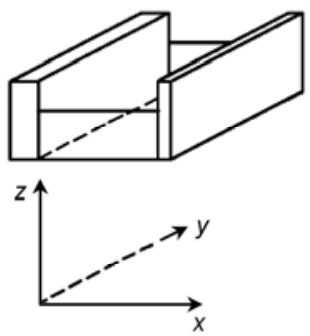

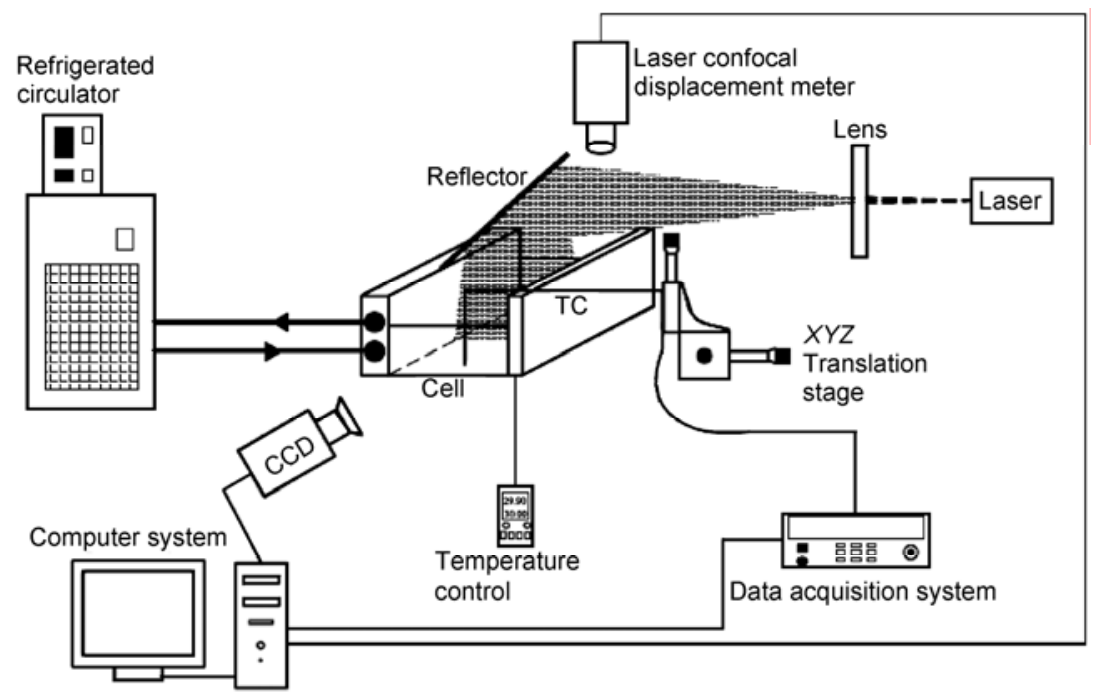

Figure 1 Schematic diagram of the experimental apparatus. 
A Dow-Corning silicone oil with a kinetic viscosity of 0.65 cSt was used as the evaporating fluid in the experiments. It was chosen not only for its desirable physical parameters, resistance to surface contamination, transparency to laser sheet for flow visualization, but also for its characteristics of evaporating under atmosphere. Another two Dow-Corning silicone oils with the viscosity of $1.5 \mathrm{cSt}$ and $5 \mathrm{cSt}$, which rarely evaporated at atmosphere, were also applied to comparing the influence of evaporation effect.

\section{Results and discussion}

To suppress the influence of Rayleigh convection existing in the ground experiments, the depth of the liquid layer must be as thinner as possible. In our experiments, the depths of the liquid layer were set to be smaller than $2.0 \mathrm{~mm}$.

As we know, both the side wall effect and the meniscus influence the stability of the flow. Therefore, we chose the middle area of the liquid layer ranging from $x=0.01 \mathrm{~m}$ to $x$ $=0.03 \mathrm{~m}$ at the streamwise direction, which was $0.01 \mathrm{~m}$ away from the two aluminum wall, to perform the measurements of the evaporating rates, temperature profiles and flow fields.

\subsection{Average evaporating rate at the interface}

The height of the interface (or the surface position) was measured by the laser co-focal displacement meter. The change of interface height could reflect the average evaporating rate when the flow in the liquid layer was steady. Figure 2 shows the variation of the measuring height of interface at one surface position during evaporation process when the temperature difference was relatively large. It could be seen that the height of interface decreased linearly during the measuring period of $2 \mathrm{~min}$, which could be considered to be a representation of steady flow. Therefore, it was feasible to use the height change to reflect the average

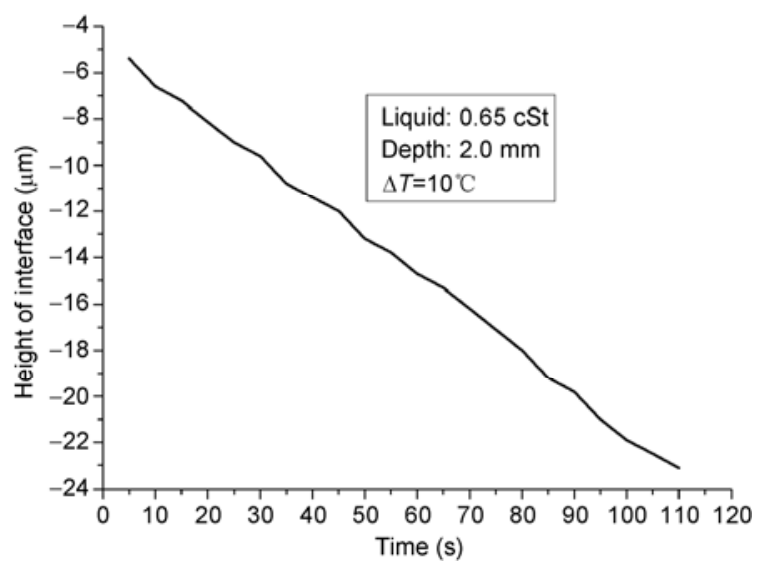

Figure 2 Change of the interface height during the evaporation process when $\Delta T=10^{\circ} \mathrm{C}$.

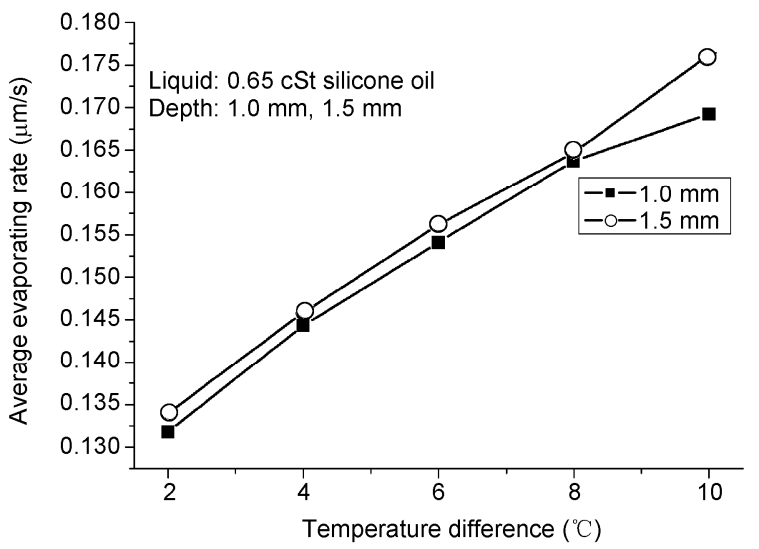

Figure 3 Total average evaporating rates along the interface for different $\Delta T$.

evaporating rate in our experiment.

Total average evaporating rates of the $0.65 \mathrm{cSt}$ silicone oil in thermocapillary flows were measured within different external horizontal temperature differences. The total average evaporating rates versus the temperature differences $(\Delta T)$ are presented in Figure 3.

The total average evaporating rate increases with the imposed temperature difference increasing. This is mainly because that the corresponding interfacial temperature of the liquid layer grows with the increase of $\Delta T$. We also found that the total average evaporating rate was bigger in deeper liquid layer. When the height of the liquid layer increased, the ability of transporting heat of the liquid layer would be enhanced and the mean interfacial temperature was increased. Generally, increasing the temperature will also enhance the average evaporating rate.

Actually, the average evaporating rate at different positions of the liquid layer will be a bit different because of the difference of the interfacial temperatures. Figure 4 shows the average evaporating rates at different streamwise positions. Note that our measurements were performed under relatively low temperature differences. And the interface of the liquid layer might be considered to be non-deformable

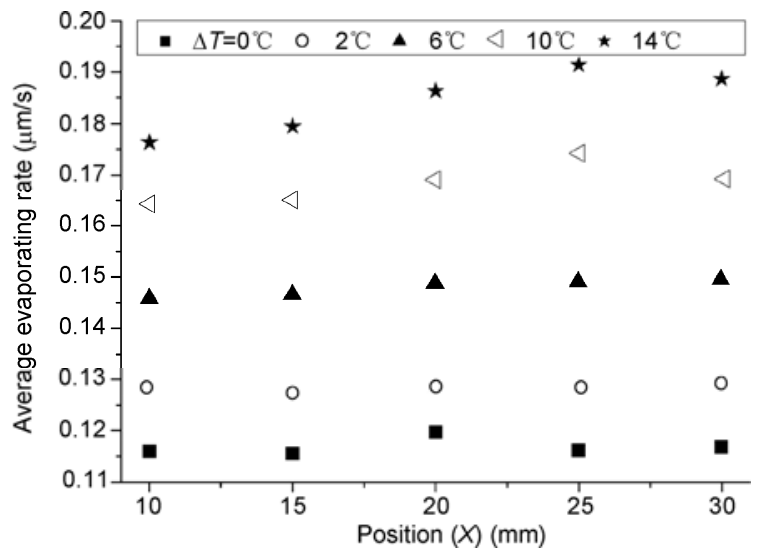

Figure 4 Average evaporating rates at different positions for different $\Delta T$. 

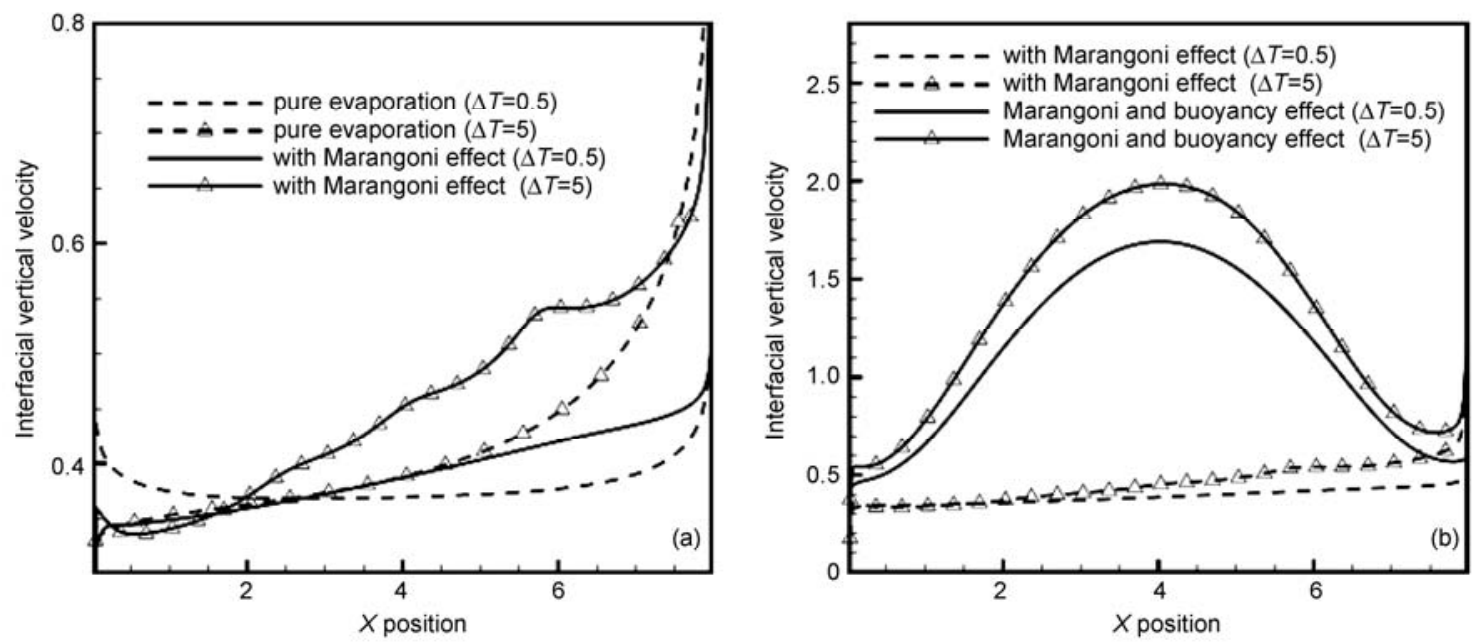

Figure 5 Evaporating rates calculated by numerical analysis. (a) Pure Marangoni effect; (b) combined Marangoni-buoyancy.

under this condition.

It can be seen that the average evaporating rate grows from $x=0.01 \mathrm{~m}$ to $x=0.03 \mathrm{~m}$, i.e. from the cold side to the hot side, when $\Delta T$ is small. However when $\Delta T$ increases to $10-14^{\circ} \mathrm{C}$, there will be a maximum average evaporating rate in the middle of the interface, at $x=0.025 \mathrm{~m}$. In fact, there is always unavoidable influence of buoyancy convection in the ground experiments. The pure thermocapillary convection influences the average evaporating rate along the interface when the temperature difference is low. While at higher temperature difference, the coupling of the thermocapillary convection in the liquid layer and the buoyancy convection in the upper air introduces the biggest concentration gradient at the middle interface, which accelerates the average evaporating rate. Comparing with the numerical results ${ }^{[16]}$, as shown in Figure 5, it could be found that the two results are consistent.

\subsection{The temperature profile at the interface}

A thermocouple was immersed just below the interface of the liquid layer to measure the temperature, and then it was taken out for the next measurement. Note that the immersing process of the thermocouple was monitored by a zoomed CCD camera to determine the interface accurately. The interfacial temperature profiles of a $2.0 \mathrm{~mm}$ deep 0.65 cSt silicone oil layer under different $\Delta T$ are shown in Figure 6.

In Figure 6, the local temperature profiles are approximately linear, i.e. the selective measuring region is stable. Out of the measuring region, we can infer that the temperature profiles are not linear. It can also be seen that the external imposed temperature gradients are always bigger than the local interfacial temperature gradients, and the difference between them will augment with the $\Delta T$ increasing. Actually, the difference of the two temperature gradients testifies the existence of the thermocapillary flow, and reflects the co-influence of the evaporation effect and thermocapillary convection. When $\Delta T$ is small $\left(0,2^{\circ} \mathrm{C}\right)$, thermocapillary convection is weaker than the evaporation effect, which takes the heat energy out of the liquid and reduces the interfacial temperature. As a result, all the five interfacial temperatures are smaller than the imposed temperatures. If the imposed temperature increases $\left(4-14^{\circ} \mathrm{C}\right)$, thermocapillary convection will be strengthened to bring more heat energy from the hot wall to the cold wall, which increases the temperature close to the cold wall. On the other hand, the evaporation effect takes more heat from the hot side of the layer to reduce the temperature. Eventually, the coupling of the evaporation effect and thermocapillary convection smoothes the interfacial temperature profiles.

To study the influence of the layer depth on the interfacial temperature, we measured the interfacial temperature profiles under two different depths of the liquid layer, $1.0 \mathrm{~mm}$ and 2.0 $\mathrm{mm}$, in $0.65 \mathrm{cSt}$ silicone oil. The comparison of the two interfacial temperature profiles is presented in Figure 7.

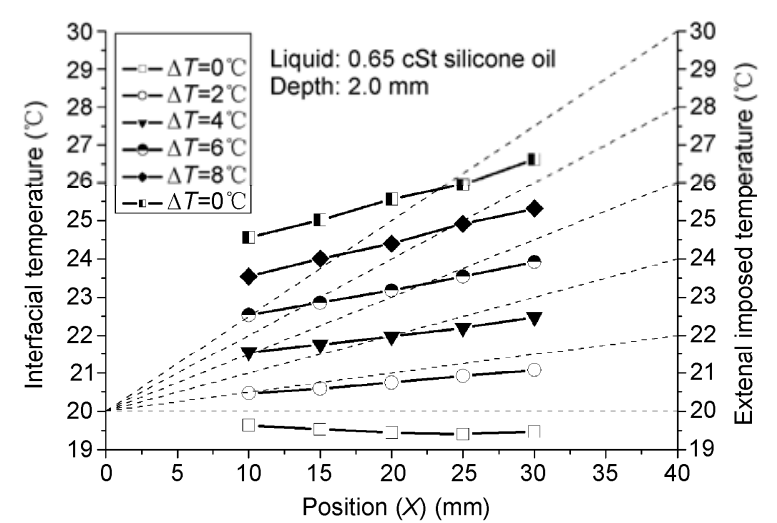

Figure 6 The interfacial temperature profiles in $0.65 \mathrm{cSt}$ sicone oil at different $\Delta T$ (depth of the liquid layer: $2.0 \mathrm{~mm}$ ). Dash line, external imposed temperature gradients. 


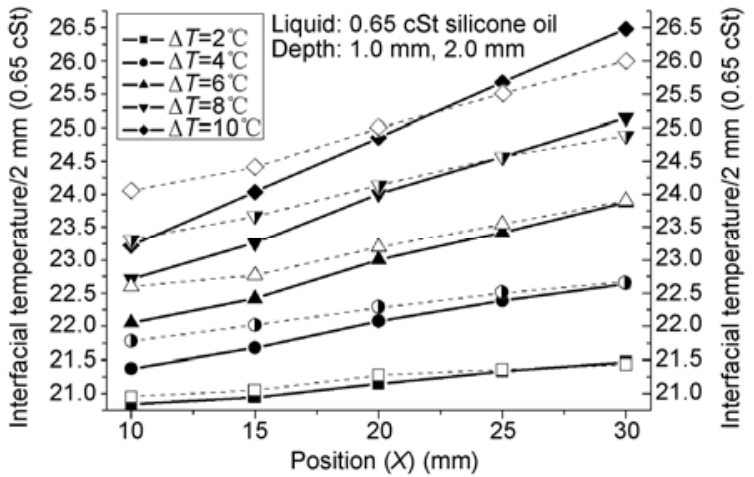

Figure 7 The interfacial temperature profiles in $0.65 \mathrm{cSt}$ silicone oil at varying depth under different $\Delta T$. Solid line, $1.0 \mathrm{~mm}$ in depth; dash line: $2.0 \mathrm{~mm}$ in depth.

As soon as the temperature gradient is established, there will be a thermocapillary flow from the hot wall to the cold wall. The investigation of the flow fields shows that a deeper layer will increase the strength of the flow. This means that more heat energy is brought from the hot side to the cold side in a deeper liquid layer. Although the evaporating rate is bigger in a deeper layer, the synchronous increase of thermocapillary flow could bring more heat energy to supply the loss of heat taken by evaporation effect. In Figure 7, we can see that the interfacial temperatures of a deep layer in $2.0 \mathrm{~mm}$ are always higher than that of a deep layer in 1.0 $\mathrm{mm}$ when $\Delta T$ is small $\left(2^{\circ} \mathrm{C}, 4^{\circ} \mathrm{C}, 6^{\circ} \mathrm{C}\right)$. As $\Delta T$ increases to $8-10^{\circ} \mathrm{C}$, thermocapillary flow is strengthened to transport more heat to the cold side at the interface. Then the evaporating rate of the $2.0 \mathrm{~mm}$ deep layer is big enough to reduce the interfacial temperature neighbouring the hot wall; meanwhile, the return flow from the cold side brings more heat to reduce the temperature close to the hot wall. The interaction of the three effects smoothes the interfacial temperature profiles in the deeper liquid layer.

To verify the influence of evaporation effect on the temperature profiles, experiments were repeated in a $1.5 \mathrm{cSt}$ liquid layer. The interfacial temperature comparison of 0.65 cSt and $1.5 \mathrm{cSt}$ silicone oil is given in Figure 8.

When $\Delta T$ is small, the temperatures in $0.65 \mathrm{cSt}$ are lower than that in $1.5 \mathrm{cSt}$ because of the evaporation effect in 0.65 cSt. The temperature differences between the two liquids close to the hot wall are bigger than that near the cold wall, because the evaporating rate is higher on the hot side. As $\Delta T$ increases, thermocapillary convection begins to play a more important role than evaporation effect. As a result, although the evaporating rate in $0.65 \mathrm{cSt}$ augments more to reduce the temperature neighbouring the hot wall, in the meantime, the thermocapillary convection is strong enough to supply the heat lost by evaporation. On the cold side, more heat is brought from the hot side than that in $1.5 \mathrm{cSt}$ owing to the lower flow velocity in $1.5 \mathrm{cSt}$, because its viscosity is larger than $0.65 \mathrm{cSt}$. The reflection in Figure 8 is that the slope of the temperature profile in $0.65 \mathrm{cSt}$ is smaller than that in 1.5

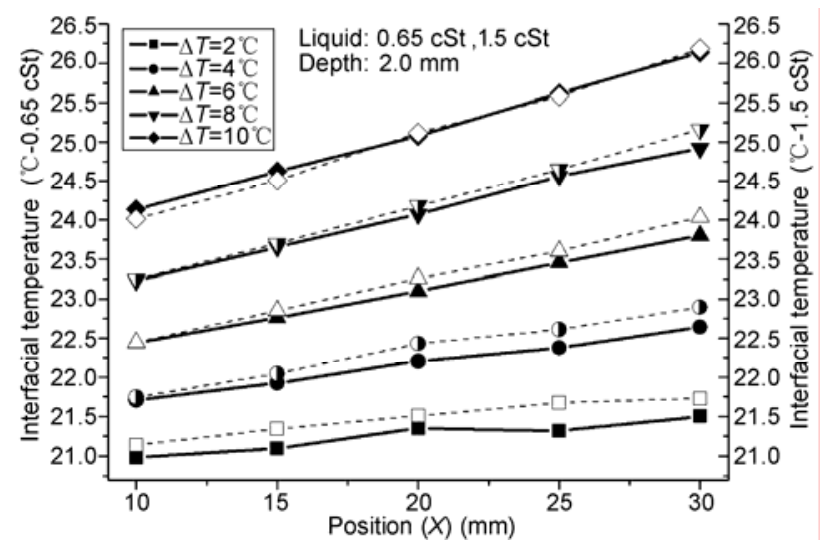

Figure 8 Interfacial temperature profiles in $0.65 \mathrm{cSt}$ and $1.5 \mathrm{cSt}$ silicone oil at different $\Delta T$ (depth of the liquid layer: $2.0 \mathrm{~mm}$ ). Solid line, $0.65 \mathrm{cSt}$; dash line, $1.5 \mathrm{cSt}$.

cSt silicone oil.

\subsection{Flow visualization}

Evaporation is also influenced by thermocapillary flow besides the interfacial temperature field. The faster thermocapillary flow is, the more heat will be transferred, and the more fluid will possibly be evaporated. Based on the thoughts, we investigated the flow fields in thin evaporating liquid layer.

Thermocapillary convection may induce an interfacial flow from the hot wall to the cold wall. For conservation of mass, there will be a reversed return flow at the bottom of the liquid layer. The flow patterns exhibit great dependence on physical parameters and $\Delta T$. Based on this, we used the PIV method to obtain the flow structures in a streamwise transverse plane of the liquid layer (the $X Z$ plane in coordinates). The dependence of flow patterns on temperature differences in a $2.0 \mathrm{~mm}$ deep layer for $0.65 \mathrm{cSt}$ and $5 \mathrm{cSt}$ silicone oil is given in Figures 9 and 10, respectively.

There are three major flow patterns of $0.65 \mathrm{cSt}$ in the observation: A long unicellular roll perpendicular to the temperature gradient appears to run through the whole test cell when $\Delta T$ is small; a steady multicellular flow appears to be a behaviour of the travelling wave when $\Delta T$ increases; a
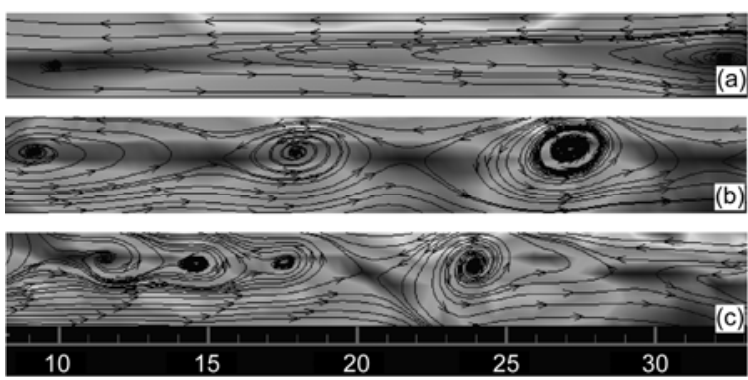

Figure 9 Flow fields of $0.65 \mathrm{cSt}$ silicone oil visualized by PIV (scale $X: Z=1: 2$ ). (a) $\Delta T=2^{\circ} \mathrm{C}$; (b) $\Delta T=6{ }^{\circ} \mathrm{C}$; (c) $\Delta T=14^{\circ} \mathrm{C}$. 

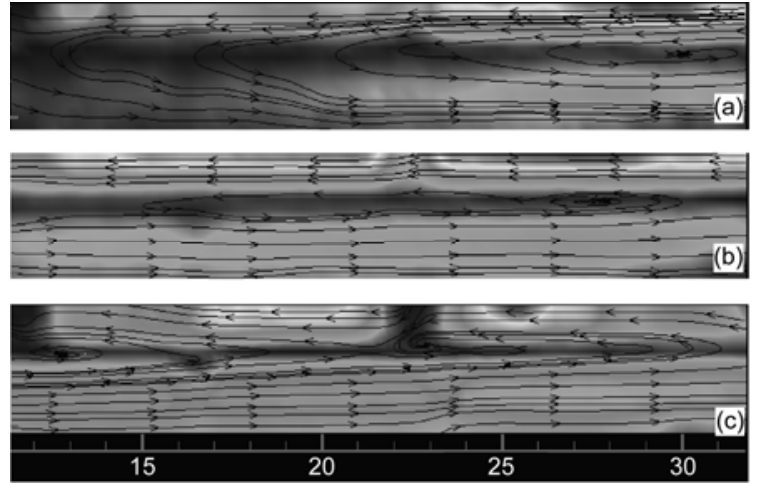

Figure 10 Flow fields of $5 \mathrm{cSt}$ silicone oil visualized by PIV (scale $X: Z=1: 2$ ). (a) $\Delta T=2{ }^{\circ} \mathrm{C}$; (b) $\Delta T=6^{\circ} \mathrm{C}$; (c) $\Delta T=14^{\circ} \mathrm{C}$

disturbed flow field is observed when $\Delta T$ continually increases, which is possibly accounted for by buoyancy convection.

Repeated investigations were performed in a $5 \mathrm{cSt}$ layer of $2.0 \mathrm{~mm}$. We found that the flow patterns were independent of $\Delta T$, always behaving to be a unicellular flow. The in creasing temperature difference just sped up the thermocapillary flow.

\section{Conclusions}

Experiments were performed to study the coupling phenomena of evaporation effect and thermocapillary convection in a thin liquid layer with an evaporating interface open to air. Evaporating rates, interfacial temperature profiles and flow fields were measured and analyzed primarily.

(i) The total average evaporating rate augments with the increase of temperature difference and layer depth. The average evaporating rate exhibits dependence on the measuring position and temperature gradient.

(ii) Thermocapillary flow is testified by measuring the interfacial temperature profiles. Comparing the temperature profiles under two conditions: varying layer depths in 0.65 $\mathrm{cSt}$, repeated measurements in $0.65 \mathrm{cSt}$ and $1.5 \mathrm{cSt}$ layer, evaporation effect is found to influence the interfacial temperature evidently. Coupling of the evaporation effect and thermocapillary convection smoothes the slopes of temperature profiles.

(iii) The flow patterns in a $2.0 \mathrm{~mm}$ deep $0.65 \mathrm{cSt}$ layer transit from a unicellular flow, via a multicellular flow, to a disturbed flow with the temperature difference increasing. Only the unicellular flow could be found in $5 \mathrm{cSt}$ layer under the same experimental conditions.

The experiments were conducted when the liquid layer was open to air. That is, the liquid layer was in the process of free evaporation. Recent researches have found a temperature jump at the evaporating interface $[17,18]$ during high evaporating process. New work is being carried out to control the evaporating rate of the layer. More details will be presented in the future.

This work was supported by the National Natural Science Foundation of China (Grant Nos. 10772185, 50890182), Knowledge Innovation Projects of Chinese Academy of Sciences (Grant No. KGCX-SW-409) and Institate of Mechanics, CAS.

1 Schwabe D. Marangoni effects in crystal growth melts. PhysicoChem Hydrodyn, 1981, 2: 263-280

2 Gatos H C. Semiconductor crystal growth and segregation problems on Earth and in space. Materials Processing in the Reduced Gravity Environment of Space, Elsevier, 1982

3 Smith M K, Davis S H. Instabilities of dynamic thermocapillary liquid layers. Part 1: Convective instabilities. J Fluid Mech, 1983, 132: 119-144

4 Smith M K. Instability mechanisms in dynamic thermocapillary liquid layers. Phys Fluids, 1986, 29: 3182-3186

5 Villers D, Platten J K. Coupled buoyancy and Marangoni convection in acetone: Experiments and comparison with numerical simulations. J Fluid Mech, 1992, 234: 487-510

6 Daviaud F, Vince J M. Traveling waves in a fluid layer subjected to a horizontal temperature gradient. Phys Rev E, 1993, 48: 4432-4436

7 Saedeleer C D, Garcimartin A, Lebon G, et al. The instability of a liquid layer heated from the side when the upper surface is open to air. Phys Fluids, 1996, 8: 670-676

8 Mercier J F, Normand C. Buoyant-thermocapillary instabilities of differentially heated liquid layers. Phys Fluids, 1996, 8: 1433-1445

9 Gillon P, Homsy G M. Combined thermocapillary-buoyancy convection in a cavity: An Experimental Study. Phys Fluids, 1996, 8: 2953-2962

10 Braunsfurth M G, Homsy G M. Combined thermocapillary buoyancy convection in a cavity. Part II: An experimental study. Phys Fluids, 1997, 9: 1277-1286

11 Riley R J, Neitzel G P. Instability of thermocapillary-buoyancy convection in shallow layers. Part 1: Characterization of steady and oscillatory instabilities. J Fluid Mech, 1998, 359: 143-164

12 Guo X C, Dong G F, Qiu Y. Electrical bistable characteristics of poly(phenylenesulfide) thin film deposited by thermal evaporation. Chinese Sci Bull, 2007, 52: 732-735

13 Yang $\mathrm{H} \mathrm{X}, \mathrm{Su} Z \mathrm{Z} \mathrm{H}$. Individual dispersion of synthetic imogolite nanotubes via droplet evaporation. Chinese Sci Bull, 2007, 52: 2301-2303

14 Chai A, Zhang N. Experimental study of Marangoni-Bénard convection in a liquid layer induced by evaporation. Exp Heat Transfer, 1998, 11: 187-205

15 Zhang N, Chao D F. Mechanisms of convection instability in thin liquid layers induced by evaporation. Int Comm Heat Mass Transfer, 1999, 26: 1069-1080

16 Liu R, Liu Q S, Hu W R. Marangoni-Bénard instability with the exchange of evaporation at liquid-vapor interface Chin Phys Lett, 2005, 22: 402-404

17 Liu R, Liu Q S. Vapour recoil effect on a vapour-liquid system with a deformable interface. Chin Phys Lett, 2006, 23: 879-882

18 Ji Y, Liu Q S, Liu R. Coupling of evaporation and thermocapillary convection in a liquid layer with mass and heat exchanging interface. Chin Phys Lett, 2008, 25: 608-611

19 Ward C A, Duan F. Turbulent transition of thermocapillary flow induced by water evaporation. Phys Rev E, 2004, 69: 056308

20 Popov S, Melling S, Durst F, et al. Apparatus for investigation of evaporation at free liquid-vapour interfaces. Int J Heat Mass Transfer, 2005, 48: 2299-2309 